\title{
Transmembrane channel-like protein 8 as a potential biomarker for poor prognosis of hepatocellular carcinoma
}

\author{
PANPAN LU, QIANG DING, SHUPING DING, YUHUI FAN, XIN LI, DEAN TIAN and MEI LIU \\ Institute of Liver Diseases, Department of Gastroenterology, Tongji Hospital, Tongji Medical College, \\ Huazhong University of Science and Technology, Wuhan, Hubei 430000, P.R. China
}

Received July 5, 2016; Accepted April 4, 2017

DOI: $10.3892 / \mathrm{mco} .2017 .1285$

\begin{abstract}
Hepatocellular carcinoma (HCC) is an aggressive malignant tumor and the third leading cause of cancer-related mortality worldwide. Transmembrane channel-like protein 8 (TMC8) is reported to play a major role in several aspects of human pathophysiology, such as ion channel permeability, human papillomavirus infection and skin cancer; however, its role in HCC has not been fully elucidated. The aim of the present study was to investigate the expression levels of TMC8 in 146 pairs of liver cancer samples and adjacent non-tumorous samples using immunohistochemistry. Reverse transcription-quantitative polymerase chain reaction analysis was used to confirm the results. The association between TMC8 expression and clinicopathological characteristics, including overall survival, was analyzed. The results indicated that the expression of TMC8 was significantly upregulated in HCC tissues and associated with metastasis and hepatitis B virus infection. According to the analysis of the overall survival using Cox proportional hazard regression model, higher expression of TMC8 was associated with a poorer prognosis and the overexpression of TMC8 was an independent risk factor for HCC. By contrast, HBsAg did not significantly affect the survival of HCC patients. These results suggest that the overexpression of TMC8 in HCC predicts poor prognosis and may be a potential biomarker for this type of cancer.
\end{abstract}

\section{Introduction}

Hepatocellular carcinoma (HCC) is one of the most common malignancies and the third leading cause of cancer-related mortality worldwide (1). Moreover, HCC is highly malignant due to its rapid development and metastasis. The morbidity and

Correspondence to: Dr Mei Liu, Institute of Liver Diseases, Department of Gastroenterology, Tongji Hospital, Tongji Medical College, Huazhong University of Science and Technology, Wuhan, Hubei 430000, P.R. China

E-mail: fliumei@tjh.tjmu.edu.cn

Key words: hepatocellular carcinoma, transmembrane channel-like protein 8 , expression, survival, prognosis mortality of HCC increase annually, which make early diagnosis and treatment crucial (2). However, several patients at diagnosis have already missed the opportunity for surgery, as they present with advanced disease, while radiotherapy and chemotherapy have not been found to be effective for HCC (3). Therefore, it is crucial to identify predictive biomarkers of HCC.

A member of the family of transmembrane channel-like (TMC) proteins, TMC8 (4), is located in the endoplasmic reticulum (ER) and reported to be expressed in a number of tissues, including the skin, breast, uterus, testes, lung and ovary (5). TMC8 also plays a role in the immune system and is expressed in several immune cells, such as T and B lymphocytes, natural killer cells, endothelial cells, bone marrow myeloid cells and dendritic cells (6). A recent study demonstrated that TMC8 limits the activation of $\mathrm{Ca}^{2+}$-dependent and volume activated whole-cell currents, probably by controlling compartmentalized intracellular $\mathrm{Ca}^{2+}$ signaling (7). TMC8 also plays a crucial role in controlling cell survival by inducing tumor necrosis factor (TNF)- $\alpha$-and TNF-related apoptosis-inducing ligand-dependent apoptosis (8). Mutations of the TMC8 gene may promote human papillomavirus (HPV) transcription and suppress the nuclear factor (NF)- $\mathrm{B}$ signaling pathways, which in turn may lead to a high likelihood of HPV infection and malignant transformation (9).

Defects of the TMC8 gene have been reported to be associated with the development of anogenital cancer $(10,11)$, epidermodysplasia verruciformis (EV) (12-15) and HPV-related head and neck squamous cell cancer (16), all of which are virus-associated diseases. Chronic hepatitis B virus (HBV) infection has been considered as the major risk factor for HCC development (17). Approximately 55\% of HCCs worldwide are HBV-related and this proportion is significantly higher in Asia (17). It was hypothesized that TMC8 may also play a role in HCC, which is also a virally-driven cancer. The expression of TMC8 in HCC tissues has not been fully elucidated to date. The aim of the present study was to investigate the association between TMC8 and HCC and identify a potential novel therapeutic target for HCC.

\section{Patients and methods}

Patients and samples. A total of 146 pairs of liver cancer samples and adjacent non-tumorous samples were collected from patients undergoing surgical resection of HCC at the Tongji Hospital 
of Huazhong University of Science and Technology (Wuhan, China) between February 2013 and March 2016. None of the patients received any preoperative chemotherapy or radiation, and they were monitored postoperatively until June 10, 2016. The detailed clinicopathological characteristics are listed in Table I. Informed consent was obtained from all the patients. The study was performed according to the guidelines of the Ethics Committee of Tongji Hospital and in accordance with the ethical standards of World Medical Association Declaration of Helsinki.

RNA extraction and reverse transcription quantitative polymerase chain reaction ( $R T-q P C R)$. A total of 19 pairs of tissue samples were randomly selected from the 146 pairs. RNA extraction and RT-qPCR were performed as previously described (18).

Tissue microarray and immunohistochemistry (IHC). All 146 pairs of tissue samples were fixed in formalin and embedded in paraffin. The diagnosis was confirmed by at least two liver cancer pathologists. The tissue microarray (TMA) was prepared by Zuocheng Biotech (Shanghai, China). A rabbit monoclonal antibody against TMC8 (catalog no. 4605, Signalway Antibody LLC, Maryland, USA) was used as the primary antibody. The TMA was incubated with the primary antibody (dilution, 1:100) overnight at $4^{\circ} \mathrm{C}$ and then incubated with the secondary anti-rabbit monoclonal antibody (dilution, 1:400; catalog no. GB23303-1; Guge Biotechnology Co., Ltd., Wuhan, China) for $10 \mathrm{~min}$ at $37^{\circ} \mathrm{C}$. The specific binding of the secondary to the primary antibody was visualized using a DAB Horseradish Peroxidase Color Development kit (GBCBIO Technologies Inc., Guangzhou, China). The IHC results were scored as follows: 0 , the expression of TMC8 in the cancer samples was not higher compared with that in the adjacent non-tumorous tissues; 1 , the expression of TMC8 in cancer samples was higher.

Statistical analysis. All data were analyzed using SPSS software, version 17.0 (SPSS Inc., Chicago, IL, USA). The paired t-test was used to compare the difference in TMC8 expression levels between paired groups. The Chi-squared test or Fisher's exact test were used to analyze the association between clinicopathological characteristics and TMC8 expression. The survival curve was constructed using the Kaplan-Meier method. The Cox proportional hazard regression model was used to analyze the risk factors for HCC. All the tests were two-sided, and $\mathrm{P}<0.05$ was considered to indicate statistically significant differences.

\section{Results}

Results of TMC8 expression in HCC tissue samples with $R T$ - $q P C R$. The expression of TMC8 was investigated in 19 pairs of liver cancer samples and adjacent non-tumorous samples with RT-qPCR. The results revealed that the TMC8 expression in liver cancer samples was significantly higher compared with that in their adjacent non-tumorous samples $(\mathrm{P}<0.0001$; Fig. 1).

IHC results of TMC8 expression in HCC tissues. Since TMC8 is also widely expressed in immune cells, we attempted to determine whether the expression of TMC8 on IHC was attributable to tumor cells or immune infiltrates. It was concluded that TMC8 was expressed by the HCC cells and the results were reviewed by two pathologists. According to the IHC results, of the 146 cancer samples, 121 (82.9\%) exhibited higher expression of TMC8, which was consistent with the results of RT-qPCR. As shown in Fig. 2, the TMC8 protein was mainly located in the cytoplasm of the cells, in accordance with previous reports. In addition, TMC8 was also occasionally found in the nuclei of liver cancer cells in a proportion of cases.

Association between TMC8 expression and clinicopathological characteristics of HCC patients. The association between TMC8 expression and the clinicopathological characteristics of the 146 HCC patients was analyzed. As shown in Table I, the expression of TMC8 exhibited a positive correlation with metastasis $(\mathrm{P}=0.016)$ and HBsAg positivity $(\mathrm{P}=0.03)$. There were no significant differences in gender, age, tumor size, tumor differentiation or $\alpha$-fetoprotein (AFP) levels $(\mathrm{P}>0.05)$.

Association of TMC8 expression with overall survival (OS) of HCC patients. The patients were followed up postoperatively and the 3-year OS of 47 patients (TMC8 high expression, $\mathrm{n}=35$ and low expression, $\mathrm{n}=12$ ) was assessed using the Kaplan-Meier survival analysis. As shown in Fig. 3, the OS of HCC patients with high expression of TMC8 was significantly poorer compared with that of patients with low expression of TMC8 ( $\mathrm{P}=0.033)$.

Association of HBsAg with the OS of HCC patients. As previously mentioned, TMC8 has been reported to play a role in certain virus-associated cancers and HBV infection accounts for the occurrence of most HCCs. It was observed that TMC8 expression exhibited a positive correlation with HBsAg positivity. To elucidate the effects of HBsAg on the OS of HCC patients, Kaplan-Meier survival analysis was used. As shown in Fig. 4, the OS of $\mathrm{HCC} \mathrm{HBsAg}{ }^{+}$patients $(n=40)$ was not significantly different from that of $\mathrm{HBsAg}^{-}$patients $(\mathrm{n}=7)(\mathrm{P}=0.059)$.

Cox proportional hazard regression model for confirming TMC8 as an independent risk factor for HCC. In order to confirm whether TMC8 was an independent risk factor for the OS of HCC, the Cox proportional hazard regression model was adopted. The result suggested that TMC8 expression was an adverse prognostic factor for HCC [95\% confidence interval (CI): 0.129-0.913; $\mathrm{P}=0.032]$. However, HBsAg was not correlated with the OS of HCC patients (95\% CI: 0.779-6.948; $\mathrm{P}=0.131$ ). Therefore, based on these results, it was concluded that the overexpression of TMC8 was an independent risk factor for the OS of HCC patients.

\section{Discussion}

HCC is a highly fatal disease, with high incidence and mortality rates. Therefore, early diagnosis is crucial. The aim of the present study was to evaluate the association between TMC8 expression and HCC, with the aim of identifying a novel potential early diagnostic and prognostic biomarker.

Previous studies suggested that the expression of TMC8 is closely associated with EV, a genodermatosis characterized 
Table I. Correlation between the expression of transmembrane channel-like protein 8 and the clinicopathological characteristics of hepatocellular carcinoma patients.

\begin{tabular}{|c|c|c|c|c|}
\hline Variables & $\begin{array}{c}\text { Samples } \\
(\mathrm{n}=146 \text { pairs })\end{array}$ & $\begin{array}{l}\text { High expression } \\
(\mathrm{n}=121 \text { pairs })\end{array}$ & $\begin{array}{l}\text { Low expression } \\
\quad(\mathrm{n}=25 \text { pairs })\end{array}$ & P-value \\
\hline Age (years) & & & & 0.353 \\
\hline$\leq 55$ & 88 & 75 & 13 & \\
\hline$>55$ & 58 & 46 & 12 & \\
\hline Gender & & & & 0.41 \\
\hline Male & 117 & 95 & 22 & \\
\hline Female & 29 & 26 & 3 & \\
\hline Tumor size $(\mathrm{cm})$ & & & & 0.209 \\
\hline$\leq 5$ & 108 & 87 & 21 & \\
\hline$>5$ & 38 & 34 & 4 & \\
\hline Tumor differentiation & & & & 0.138 \\
\hline High & 15 & 10 & 5 & \\
\hline Poor or moderate & 131 & 111 & 20 & \\
\hline Metastasis & & & & 0.016 \\
\hline Yes & 73 & 66 & 7 & \\
\hline No & 73 & 55 & 18 & \\
\hline$\alpha$ fetoprotein $(\mathrm{ng} / \mathrm{ml})$ & & & & 0.55 \\
\hline$\leq 400$ & 78 & 66 & 12 & \\
\hline$>400$ & 68 & 55 & 13 & \\
\hline HBsAg & & & & 0.03 \\
\hline Positive & 123 & 106 & 17 & \\
\hline Negative & 23 & 15 & 8 & \\
\hline
\end{tabular}

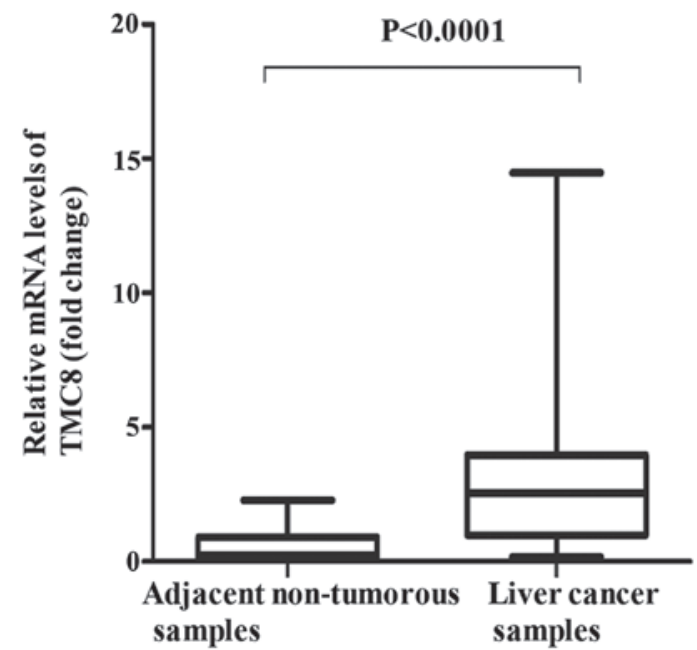

Figure 1. Expression of transmembrane channel-like protein 8 (TMC8) in 19 pairs of liver cancer samples and their adjacent non-tumorous samples measured by reverse transcription-quantitative polymerase chain reaction analysis. The expression level of TMC8 in the non-tumorous samples was significantly lower compared with that in the liver cancer samples $(\mathrm{P}<0.0001)$.

by an immune defect and the development of skin cancer due to its selective susceptibility to HPV $(12,13,19)$. It has been confirmed that EVER2 loss activates the c-Jun N-terminal kinase/activating protein-1 (AP-1) signaling pathway, which may contribute to the overexpression of interleukin (IL)-6 (9).
IL-6 may promote the formation of an inflammatory microenvironment and accelerate the development of cancer (20). AP-1 is a transcription factor playing a key role in the life cycle of HPV (19). Furthermore, EVER2 loss also represses the transcriptional activity of $\mathrm{NF}-\kappa \mathrm{B}(9)$. All these results indicate that TMC8 defects induce HPV transcription but suppress $\mathrm{NF}-\kappa \mathrm{B}$, ultimately promoting the progression of EV to skin cancer (9). Similarly, the mutation of TMC8 exacerbates HPV infection and eventually leads to the development of cervical cancer $(11,21)$.

TMC8 controls receptor-mediated $\mathrm{Ca}^{2+}$ release from ER stores, which counteracts the activation of anoctamin 1 (Ano1) (7). Anol is a $\mathrm{Ca}^{2+}$-activated $\mathrm{Cl}^{-}$channel, which is associated with head and neck cancer, often caused by HPV infection (22-24). A recent report suggested that the upregulated $\mathrm{Zn}^{2+} / \mathrm{Ca}^{2+}$ signaling and activation of Anol may be associated with cancers caused by the mutation of TMC8 (7). TMC8 also inhibits volume-activated $\mathrm{Cl}^{-}$currents, suggesting that TMC8 may be associated with volume regulated anion channel (VRAC) (7,25). VRAC is a volume regulator, and also affects several other functions, such as apoptosis, cell proliferation and migration (26). In addition, TMC8 may promote TNF- $\alpha$-induced apoptosis by binding TNF receptor type 1-associated DEATH domain protein through the TMC-containing region (8).

As mentioned before, TMC8 was previously considered to be a tumor suppressor. In order to elucidate the association 

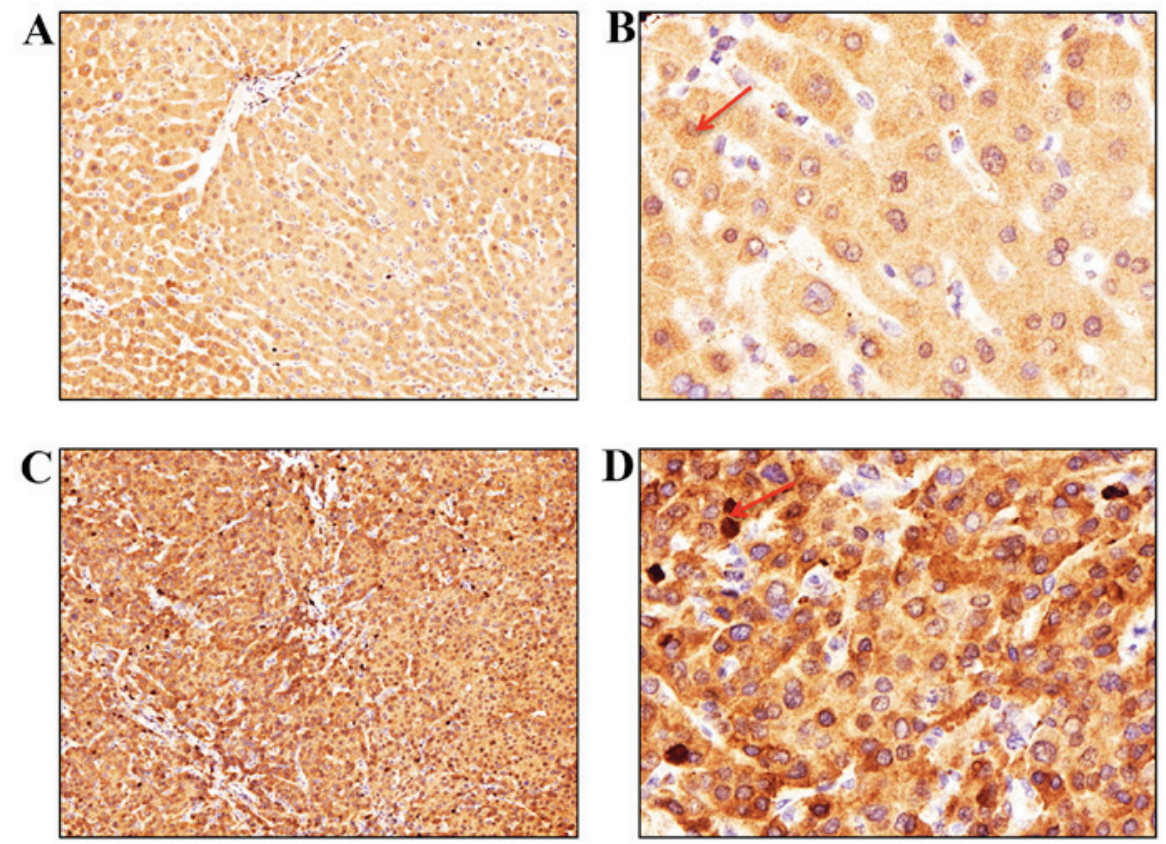

Figure 2. Expression of transmembrane channel-like protein 8 (TMC8) in 146 pairs of liver cancer and adjacent non-tumorous tissues by immunohistochemistry. (A and B) expression of TMC8 in adjacent non-tumorous tissue; (C and D) expression of TMC8 in liver cancer tissue. TMC8 is mainly expressed in the cytoplasm. The red arrows indicate occasional nuclear expression of TMC8. The expression of TMC8 in the liver cancer tissue was significantly higher compared with that in adjacent non-tumorous tissue. Original magnification: (A and C), x100; (B and D), x400.

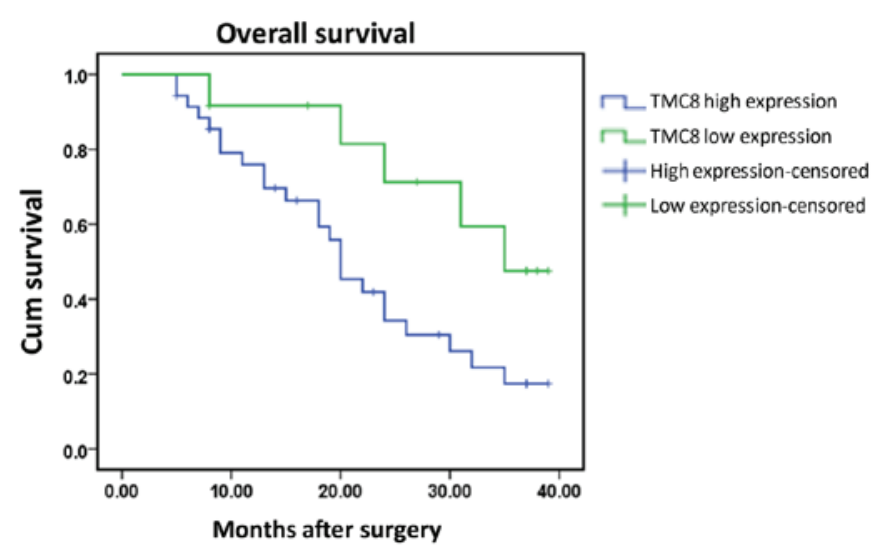

Figure 3. Kaplan-Meier survival curves according to transmembrane channel-like protein 8 (TMC8) expression in patients with hepatocellular carcinoma. Patients with high expression of TMC8 exhibited a significantly shorter survival time compared with that of patients with low expression of TMC8 $(\mathrm{P}<0.05)$

between TMC8 and HCC, the expression of TMC8 was investigated in 146 pairs of tissues. According to the IHC results in the present study, the expression of TMC8 in liver cancer tissues was higher compared with that in adjacent non-tumorous samples. The positive rate was $82.9 \%$. As TMC8 may be also expressed by immune cells, it must first be elucidated whether the observed expression of TMC8 is in the tumor cells or the immune infiltrates. All the IHC images were carefully examined and there was no obvious TMC8 expression in the stroma of HCC tissues, such as the portal area. Thus, the observed expression of TMC8 was indeed in the tumor cells rather than the immune cells. These results were confirmed by two pathologists. In fact, despite several immunocyte-related reports on TMC8 function, TMC8 has been reported to be

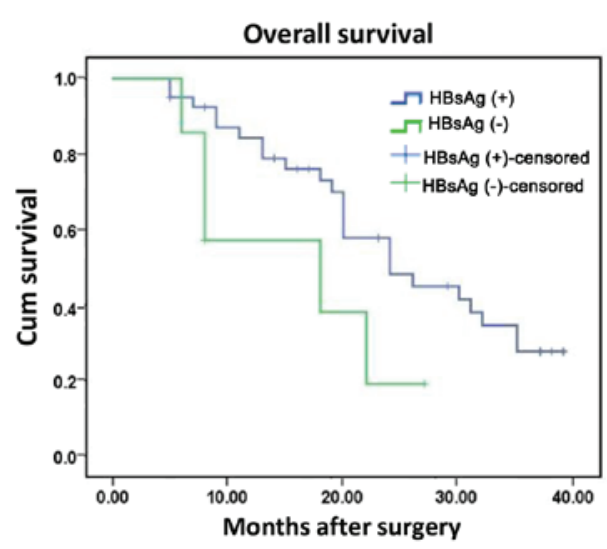

Figure 4. Kaplan-Meier survival curves according to HBsAg status in patients with hepatocellular carcinoma. There was no significant difference in overall survival between $\mathrm{HBsAg}^{+}$and $\mathrm{HBsAg}^{-}$patients $(\mathrm{P}>0.05)$.

widely expressed in a number of tissues, such as skin and breast epithelia (5). In addition, TMC8 expression was also measured by RT-qPCR and the results were in accordance with the IHC results. Therefore, TMC8 was expressed in liver cancer tissues and its overexpression may be a potential biomarker of HCC. It remains unclear why the expression of TMC8 in HCC differed from previously reported results. In fact, the number of studies investigating the role of TMC8 in tumors is limited, their results are inconclusive, and the majority investigate HPV-related cancers; thus, they may be not be considered as comprehensive. In addition, apart from the cytoplasmic expression of TMC8, nuclear expression was also observed in some cases on IHC, which, to the best of our knowledge, has not been previously mentioned. It could not be determined whether this was an incidental occurrence and 
further studies are required to elucidate the significance of this finding.

The association between TMC8 expression and the clinicopathological characteristics of the HCC patients was also analyzed. There were significant differences in metastasis $(\mathrm{P}<0.05)$ and HBsAg positivity $(\mathrm{P}<0.05)$ between the high and low TMC8 expression groups, indicating that high TMC8 expression was closely associated with metastasis and HBV infection. To the best of our knowledge, metastasis predicts an unfavourable prognosis and HBV infection may gradually lead to the occurrence of HCC; both suggest that TMC8 may be an effective index for evaluating the progression and prognosis of HCC. There was no significant difference in gender, age, tumor size, tumor differentiation or AFP levels between the high and low expression groups $(\mathrm{P}>0.05)$.

The survival of 47 patients after 3 years of follow-up was evaluated using the Kaplan-Meier survival analysis. Among these patients, 35 exhibited high expression and 12 exhibited low expression of TMC8. The result of the survival curve indicated that patients with high expression of TMC8 had a significantly lower OS rate compared with patients who had low expression of TMC8 $(\mathrm{P}<0.05)$. In order to elucidate the role of HBsAg in the prognosis of HCC, a Kaplan-Meier survival analysis was performed. The result revealed no significant difference in survival between $\mathrm{HBsAg}^{+}$and $\mathrm{HBsAg}^{-}$patients ( $\mathrm{P}>0.05)$. Moreover, the Cox proportional hazards regression model was used to confirm whether TMC8 was associated with survival independently of $\mathrm{HBsAg}$. The result indicated that TMC8 was an independent risk factor for the prognosis of HCC $(\mathrm{P}<0.05)$, while HBsAg was not associated with the survival of HCC patients $(\mathrm{P}>0.05)$. Due to our limited follow-up data, the results may be not sufficiently detailed. However, through comprehensive analysis, it may be concluded that high expression of TMC8 is associated with survival, regardless of the HBsAg status, and is a poor prognostic marker of HCC.

In conclusion, the expression of TMC8 is upregulated in HCC and high expression of TMC8 is associated with a poor prognosis. To the best of our knowledge, this is the first study to investigate the role of TMC8 as a tumor promoter. TMC8 may be a potential biomarker of HCC. However, the exact mechanism underlying the role of TMC8 in HCC has not been fully elucidated. Therefore, further studies are required to enable a better understanding of TMC8 and its potential as a biomarker.

\section{Acknowledgements}

The present study was supported by the National Natural Science Foundation of China (grant nos. 81272657 and 81572422).

\section{References}

1. Yang JD and Roberts LR: Hepatocellular carcinoma: A global view. Nat Rev Gastroenterol Hepatol 7: 448-458, 2010.

2. El-Serag HB, Davila JA, Petersen NJ and McGlynn KA: The continuing increase in the incidence of hepatocellular carcinoma in the United States: An update. Ann Intern Med 139: 817-823, 2003.

3. Llovet JM, Ricci S, Mazzaferro V, Hilgard P, Gane E, Blanc JF, de Oliveira AC, Santoro A, Raoul JL, Forner A, et al: Sorafenib in advanced hepatocellular carcinoma. N Engl J Med 359: 378-390, 2008.

4. Keresztes G, Mutai H and Heller S: TMC and EVER genes belong to a larger novel family, the TMC gene family encoding transmembrane proteins. BMC Genomics 4: 24, 2003
5. Horton JS and Stokes AJ: The transmembrane channel-like protein family and human papillomaviruses: Insights into epidermodysplasia verruciformis and progression to squamous cell carcinoma. Oncoimmunology 3: e28288, 2014

6. Su AI, Wiltshire T, Batalov S, Lapp H, Ching KA, Block D, Zhang J, Soden R, Hayakawa M, Kreiman G, et al: A gene atlas of the mouse and human protein-encoding transcriptomes. Proc Natl Acad Sci USA 101: 6062-6067, 2004.

7. Sirianant L, Ousingsawat J, Tian Y, Schreiber R and Kunzelmann K: TMC8 (EVER2) attenuates intracellular signaling by $\mathrm{Zn} 2+$ and $\mathrm{Ca} 2+$ and suppresses activation of $\mathrm{Cl}-$ currents. Cell Signal 26: 2826-2833, 2014.

8. Gaud G, Guillemot D, Jacob Y, Favre M and Vuillier F: EVER2 protein binds TRADD to promote TNF-a-induced apoptosis. Cell Death Dis 4: e499, 2013.

9. Vuillier F, Gaud G, Guillemot D, Commere PH, Pons C and Favre M: Loss of the HPV-infection resistance EVER2 protein impairs NF-kB signaling pathways in keratinocytes. PLoS One 9: e89479, 2014.

10. Smola S: Human papillomaviruses and skin cancer. Adv Exp Med Biol 810: 192-207, 2014.

11. Castro FA, Ivansson EL, Schmitt M, Juko-Pecirep I, Kjellberg L, Hildesheim A, Gyllensten UB and Pawlita M: Contribution of TMC6 and TMC8 (EVER1 and EVER2) variants to cervical cancer susceptibility. Int J Cancer 130: 349-355, 2012.

12. Majewski S and Jablonska S: Epidermodysplasia verruciformis as a model of human papillomavirus-induced genetic cancers: The role of local immunosurveillance. Am J Med Sci 304: 174-179, 1992.

13. Landini MM, Zavattaro E, Borgogna C, Azzimonti B, De Andrea M, Colombo E, Marenco F, Amantea A, Landolfo S and Gariglio M: Lack of EVER2 protein in two epidermodysplasia verruciformis patients with skin cancer presenting previously unreported homozygous genetic deletions in the EVER2 gene. J Invest Dermatol 132: 1305-1308, 2012.

14. Orth G: Genetics of epidermodysplasia verruciformis: Insights into host defense against papillomaviruses. Semin Immunol 18: 362-374, 2006.

15. Sun XK, Chen JF and Xu AE: A homozygous nonsense mutation in the EVER2 gene leads to epidermodysplasia verruciformis. Clin Exp Dermatol 30: 573-574, 2005.

16. Liang C, Kelsey KT, McClean MD, Christensen BC, Marsit CJ, Karagas MR, Waterboer T, Pawlita M and Nelson HH: A coding variant in TMC8 (EVER2) is associated with high risk HPV infection and head and neck cancer risk. PLoS One 10: e0123716, 2015.

17. Kew MC: Epidemiology of chronic hepatitis B virus infection, hepatocellular carcinoma, and hepatitis B virus-induced hepatocellular carcinoma. Pathol Biol (Paris) 58: 273-277, 2010.

18. Ding Q, Xia Y, Ding S, Lu P, Sun L and Liu M: An alternatively spliced variant of CXCR3 mediates the metastasis of CD133+ liver cancer cells induced by CXCL9. Oncotarget 7: 14405-14414, 2016.

19. Lazarczyk M, Pons C, Mendoza JA, Cassonnet P, Jacob Y and Favre M: Regulation of cellular zinc balance as a potential mechanism of EVER-mediated protection against pathogenesis by cutaneous oncogenic human papillomaviruses. J Exp Med 205: 35-42, 2008.

20. Colotta F, Allavena P, Sica A, Garlanda C and Mantovani A: Cancer-related inflammation, the seventh hallmark of cancer: Links to genetic instability. Carcinogenesis 30: 1073-1081, 2009.

21. Wang SS, Gonzalez P, Yu K, Porras C, Li Q, Safaeian M, Rodriguez AC, Sherman ME, Bratti C, Schiffman M, et al: Common genetic variants and risk for HPV persistence and progression to cervical cancer. PLoS One 5: e8667, 2010.

22. Yang YD, Cho H, Koo JY, Tak MH, Cho Y, Shim WS, Park SP, Lee J, Lee B, Kim BM, et al: TMEM16A confers receptor-activated calcium-dependent chloride conductance. Nature 455: 1210-1215, 2008.

23. Caputo A, Caci E, Ferrera L, Pedemonte N, Barsanti C, Sondo E, Pfeffer U, Ravazzolo R, Zegarra-Moran O and Galietta LJ: TMEM16A, a membrane protein associated with calcium-dependent chloride channel activity. Science 322: 590-594, 2008.

24. Schroeder BC, Cheng T, Jan YN and Jan LY: Expression cloning of TMEM16A as a calcium-activated chloride channel subunit. Cell 134: 1019-1029, 2008.

25. Nilius B and Droogmans G: Amazing chloride channels: An overview. Acta Physiol Scand 177: 119-147, 2003.

26. Hoffmann EK, Sorensen BH, Sauter DP and Lambert IH: Role of volume-regulated and calcium-activated anion channels in cell volume homeostasis, cancer and drug resistance. Channels (Austin) 9: 380-396, 2015. 